\title{
Mitotic activity during preimplantation development of human embryos
}

\author{
M. Herbert ${ }^{1}$, J. Wolstenholme ${ }^{2}$, A. P. Murdoch ${ }^{1}$ and T. J. Butler ${ }^{3}$ \\ ${ }^{1}$ Centre for Reproductive Medicine, ${ }^{2}$ Department of Human Genetics, Royal Victoria Infirmary, \\ Newcastle Upon Tyne; and ${ }^{3}$ Department of Medical Statistics, University of Newcastle Upon Tyne, UK
}

\begin{abstract}
The rate of cell division of human embryos in vitro was estimated throughout the preimplantation period. Seventy-five human embryos were assessed by performing nuclei counts on whole mount preparations at $24 \mathrm{~h}$ intervals between day 2 and day 6 of development. The results indicate that the cell doubling time increases after the first two division cycles. The mean ( \pm SEM) doubling time between day 2 and day 6 was $31 \pm 2.51 \mathrm{~h}$. At the end of the designated culture periods, embryos were exposed to a microtubule disrupting agent, which caused the nuclei to arrest in metaphase. Thus, a profile of the mitotic activity at given stages of development was obtained. The numbers of actively dividing cells were frequently lower than anticipated, indicating that nuclei counts cannot be directly correlated with continuing mitotic activity.
\end{abstract}

\section{Introduction}

Standard practice in clinical in vitro fertilization (IVF) and embryo transfer treatment is to place embryos in the uterus during their early cleavage stages. The rate of cell division during these stages is positively correlated with embryo viability following IVF and embryo transfer (Trounson $e t$ al., 1982; Staessen et al., 1992; Margalioth et al., 1993). Cell division rate is thus used as an important indicator of embryo quality, not only to select suitable embryos for transfer but also to evaluate culture conditions.

Despite the widespread practice of in vitro culture of human embryos, little is known about the rate of cell proliferation. Data on the development rate of human embryos in vitro are based largely on the number of blastomeres visible during the early cleavage stages (Edwards et al., 1981; Trounson et al., 1982; Cummins et al., 1986). Hardy et al. (1989), Winston et al. (1991) and Dorkas et al. (1993) estimated the number of cells of blastocysts by staining and counting the nuclei. Using this approach, Hardy et al. (1989) determined the rate of cell division of blastocysts as fixed between day 5 and day 7 . Therefore, the rather sparse information on the rate of cell division in human embryos relates to either the early cleavage divisions or to the blastocyst stage. There are no data available on the rate of cell proliferation of human embryos throughout the preimplantation period.

The assessment of numbers of cells by staining and counting nuclei provides an indication of the number of cell division cycles that have occurred up to the point at which the embryos were stained and is, in this sense, historical information. There is evidence, from studies on the proliferation kinetics of normal human somatic cells, of a loss of growth potential with increasing number of cell replications in vitro. This has

Received 9 June 1994

been attributed to an increase in the fraction of noncycling cells (Smith and Braunschweiger, 1979; Ponten et al., 1983). Experiments on mouse embryos indicate that, as development proceeds, the rate of cell division slows down (Streffer et al., 1980). In addition, subpopulations of cells become asynchronous and divide at different rates during late preimplantation morphogenesis in a number of species, including humans (Johnson and Ziomek, 1982; MacQueen and Johnson, 1983; Papaioannou and Ebert, 1988; Hardy et al., 1989). Therefore, a more valid profile of the mitotic activity of human embryos needs to take into account the fraction of actively dividing cells at specific times or stages of development. This information is not available for human embryo development.

The relationship between mitotic activity and the onset of compaction and cavitation has not been investigated in humans, although it has been suggested that compaction of human embryos takes place between the fourth and fifth cleavage divisions (Edwards et al., 1981; Cummins et al., 1986; Hardy et al., 1989). Measurement of the mitotic activity in human morulae and blastocysts offers an insight into the relationship between cell proliferation and late preimplantation morphogenesis.

In this study, we set out to estimate the number of cell generation cycles that embryos undergo during each $24 \mathrm{~h}$ of culture between day 2 and day 6 of development. By counting the number of nuclei per embryo, the mean rate of cell proliferation between the early and late preimplantation stages was calculated. At the end of the designated culture period, embryos were treated with colcemid which inhibits microtubule assembly and thereby causes cells to arrest in metaphase. The number of metaphases related to the total number of nuclei provides an index of the mitotic activity at specific stages of development. 


\section{Materials and Methods}

\section{Source of embryos}

The embryos used in this study were donated for research by couples undergoing IVF treatment. Surplus embryos were allocated to the study once the IVF treatment cycle was completed. The study was approved by the Ethics Committee of Newcastle Health Authority and was licensed by the Human Fertilisation and Embryology Authority.

Superovulation was achieved by downregulation of the pituitary with the GnRH agonist, buserelin (Suprefact: Hoechst, Hounslow) and exogenous administration of either human menopausal gonadotrophin ( $\mathrm{hMG}$ ) (Pergonal: Serono, Welwyn Garden City) or FSH (Metrodin: Serono). Buserelin was administered by nasal spray in three doses of $800 \mu \mathrm{g}$ per day, until the ovarian steroids were in the postmenopausal range and no follicles were seen on the transvaginal ultrasound scan. Thereafter, hMG and FSH stimulation commenced (150-225 iu per day), while the same daily dose $(800 \mu \mathrm{g})$ of buserelin continued to be taken. Final maturation of follicles was achieved by i.m. injection of either 5000 or 10000 iu hCG (Profasi: Serono). Oocytes were retrieved by transvaginal ultrasound guided aspiration of follicles $36-38 \mathrm{~h}$ after hCG injection.

\section{Culture of gametes and embryos}

Oocytes were cultured and inseminated in $200 \mu \mathrm{l}$ droplets of medium under mineral oil (Aldrich, Dorset). The medium was Earle's balanced salt solution (EBSS; Gibco BRL, Paisley) supplemented with sodium bicarbonate $\left(25 \mathrm{mmol} \mathrm{I}^{-1}\right.$ ) (Analar grade; $\mathrm{BDH}$, Lutterworth), sodium pyruvate $\left(0.47 \mathrm{mmol} \mathrm{l}^{-1}\right)$ (Analar grade; $\mathrm{BDH})$ and $10 \%(\mathrm{v} / \mathrm{v})$ human serum albumin (HSA; 5\% solution, Albuminar-5, Armour Pharmaceuticals, Eastbourne). The culture environment was $5 \% \mathrm{CO}_{2}$ in air, $37^{\circ} \mathrm{C}$ and $99 \%$ relative humidity. Motile spermatozoa were separated from semen by centrifugation at $200 \mathrm{~g}$ for $20 \mathrm{~min}$ at $20^{\circ} \mathrm{C}$ on a discontinuous Percoll gradient (Pharmacia, Uppsala) composed of $1.5 \mathrm{ml}$ each of $90 \%$ and $45 \%$ Percoll solutions in Hepesbuffered EBSS solutions. Oocytes were inseminated $41-43 \mathrm{~h}$ after hCG injection (day 0). On day I (18-20 h after insemination) the cumulus cells surrounding the oocyte were mechanically removed either with finely drawn pipettes or needles. Normal fertilization was indicated by the presence of two pronuclei. Fertilized eggs were transferred to a fresh droplet of modified EBSS, supplemented with $15 \%(\mathrm{v} / \mathrm{v})$ of HSA under mineral oil.

\section{Embryo evaluation and selection}

Assessment of the morphology of embryos was performed on an inverted microscope at $\times 200$ magnification. The development of cleavage stage embryos was assessed by counting the number of blastomeres. The degree of cytoplasmic fragmentation and evenness of blastomere size was also noted. The classification of morulae was based on the morphological transition of the embryo from being a cluster of spherical blastomeres to being a rounded mass in which distinct divisions between the blastomeres were not visible. Embryos were

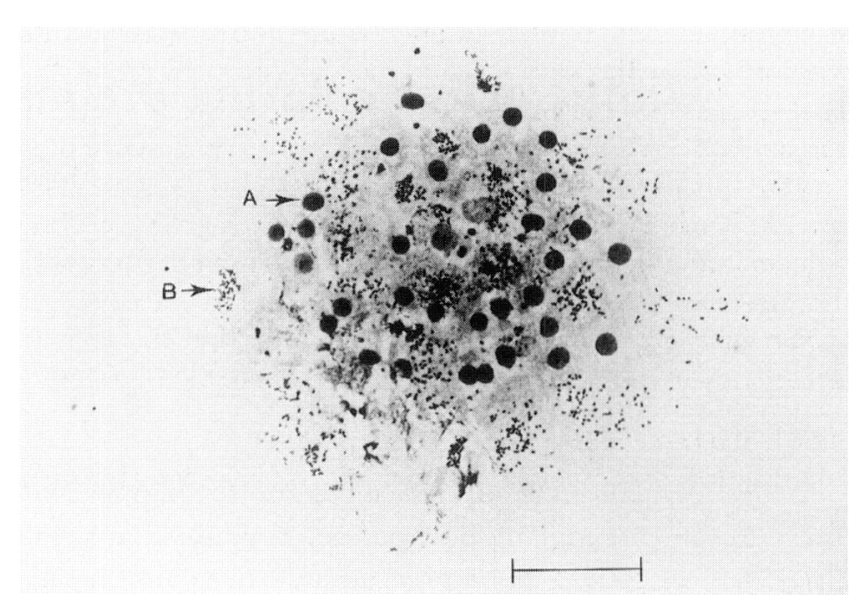

Fig. 1. Micrograph of fixed and stained expanded human blastocyst A: an interphase nucleus; B: a metaphase spread. Scale bar represents $100 \mu \mathrm{m}$.

classified as blastocysts when the blastocoelic cavity was surrounded by a distinct layer of trophoblast cells. Blastocyst expansion was subjectively assessed on the basis of volume increase and the degree of zona thinning.

On day 2 of development, $44-50 \mathrm{~h}$ after insemination, a maximum of three of the fastest growing and least fragmented embryos were selected for embryo transfer; the mean number of embryos replaced per patient was 2.6. The surplus embryos of consenting couples were then available for research. The embryos were cultured either in their original droplets of medium or they were transferred to $1 \mathrm{ml}$ of the same medium. They were cultured randomly for different periods. Heavily fragmented or degenerating embryos at each stage of culture were not included in further analysis because preparation and staining were not successful. This study therefore reflects the growth of apparently normal embryos.

\section{Staining and counting of nuclei}

At the end of the culture period, embryos were treated with $0.5 \mu \mathrm{g}$ colcemid $\mathrm{ml}^{-1}$ (Gibco BRL) for $16 \mathrm{~h}$ before being fixed and stained. Therefore, nuclei counts for each period represent the number of interphase nuclei plus the number of nuclei that entered metaphase during the $16 \mathrm{~h}$ incubation with colcemid.

Embryos were fixed in methanol and acetic acid (3:1, v:v) and stained with Giemsa stain. The zonae were removed before fixing by placing the embryos in $0.3 \mathrm{ml}$ solution of trypsin (BactoTrypsin: Gibco BRL), water and $1 \%(\mathrm{w} / \mathrm{v})$ sodium citrate (1:1:10) and left for $10 \mathrm{~min}$. An equal volume of fixative (3:1 methanol and acetic acid) was added dropwise and left for $5 \mathrm{~min}$. The embryos were then transferred to $0.3 \mathrm{ml}$ fixative and left for $30 \mathrm{~min}$ at room temperature. Finally, the embryos were placed in a 3:3:1 solution of methanol, acetic acid and water for 2-3 min, before being placed on an alcohol-cleaned glass slide. The slides were dried under a flow of warm air and placed in a $10 \%(\mathrm{w} / \mathrm{v})$ solution of Giemsa stain for 2-3 min.

Nuclei were counted at $\times 400$ magnification. Interphase nuclei stained darkly and had a distinct outline (Fig. I). There was some variation in the size of interphase nuclei but this was Downloaded from Bioscientifica.com at 04/26/2023 11:14:08AM 
mainly artefactual and due to orientation of the nucleus or its position within the embryo (i.e., the outermost nuclei in the fixed preparation were often more decondensed). Nuclear fragments were seen in some preparations; these were not counted. Of the embryos that were fixed and stained successfully, $38 \%$ could not be analysed because of extensive nuclear degeneration. Metaphase nuclei appeared as discrete clusters of chromosomes (Fig. 1); these were counted as one nucleus.

The mitotic index of embryos was calculated as the ratio of metaphases to the total number of nuclei multiplied by 100 .

mitotic index $=$

$100 \times$ (number of metaphases/total number of nuclei)

\section{Statistical analysis}

The statistical analysis was carried out using generalized linear models from the GLIM4 statistical analysis package (The GLIM System, Release 4 manual, 1993). This allowed the correct analysis of the mitotic activity, considering a binomial model with successes being the number of nuclei in mitosis out of the total number of cells in the embryo. The analysis of development rates considered normal analysis of variance techniques.

\section{Results}

A total of 216 embryos from 50 patients were allocated to this study. Fifty-five embryos were lost during the fixing process. Most of the embryos lost in the fixative were morphologically abnormal and many were undergoing degeneration. Forty-six embryos which were fixed and stained successfully could not be analysed because of nuclear fragmentation. Forty day 6 embryos were not fixed because they were in an advanced state of degeneration and it was expected that they would be lost during processing. Thus, the analysis of nuclear counts and mitotic indices were based on data from 75 embryos donated by 29 couples (range 1-5 embryos per couple). Colcemid (1.25 $\mathrm{mol} \mathrm{l}^{-1}$ ) was added to embryos at the following times after insemination: $48-50 \mathrm{~h}$ (day $2 ; n=12$ ); $72-74 \mathrm{~h}$ (day 3 ; $n=11$ ); 96-98 h (day $4 ; n=12) ; 108-110 \mathrm{~h}$ (day 5: $n=20$ ); $132-134 \mathrm{~h}$ (day $6 ; n=20$ ). Embryos were fixed following $16 \mathrm{~h}$ exposure to colcemid.

The nuclei counts and mitotic indices of individual embryos for each culture period are illustrated in Fig. 2. The total nuclei count for each embryo represents the number of interphase nuclei plus the number of metaphase nuclei. The patient origin of embryos is also indicated.

At the time of addition of colcemid, all of the day 2 and day 3 embryos were classified as being in the cleavage stages; the number of blastomeres ranged from 2 to 12 . The day 4 and day 5 embryos consisted of a mix of cleavage stage morula and blastocyst stage embryos. All of the day 6 embryos were blastocysts; five were unexpanded and 15 were expanded. The nuclei counts of embryos that were identified as morulae $(n=6)$, blastocysts $(n=12)$ and expanded blastocysts $(n=16)$ on days 4,5 and 6 are shown in Fig. 3.

The percentage of mitotic nuclei on each day was $44 \%$ on day $2,15 \%$ on day $3,18 \%$ on day $4,25 \%$ on day 5 and $25.6 \%$ on day 6. From these data, the probability of nuclei being in mitosis at the end of each culture period is illustrated in Fig. 4. The difference in the proportions of actively dividing cells on each day was not significant $(P=0.53)$, although the wide confidence limits indicate that a larger study is required to confirm this apparent effect.

The number of nuclei per embryo was plotted on a $\log _{2}$ scale against time (Fig. 5). $\log _{2}$ of the number of nuclei per embryo was taken, as it represents the number of nuclear replications undergone during the culture period. The overall rate of increase between day 2 and day 6 of 75 embryos was linear on a log scale and is expressed by the following equation:

$$
\log _{2} n=a+b t
$$

where $n$ is the number of nuclei, $t$ is the incubation time (days) and $a$ and $b$ are the regression coefficients. A regression gave values \pm SEM of $a=0.735 \pm 0.26$ and $b=0.76 \pm 0.06$. The overall doubling time of this population of embryos was $31.6 \pm 2.51 \mathrm{~h}$. This corresponds to a theoretical mean of $50 \%$ of all nuclei entering metaphase during the $16 \mathrm{~h}$ exposure to colcemid.

Again, taking $\log _{2}$ of the absolute number of nuclei per embryo as a rate of cell division over the whole culture period, there was wide variation in the numbers of nuclei per embryo within each day. To test the hypothesis that this was due to variation between donating couples, analysis of variance was performed. The analysis showed a significant person effect $(P=0.021)$ after adjustment for day effect.

The relationship between mitotic indices and late preimplantation development was investigated. The mitotic indices after $16 \mathrm{~h}$ exposure to colcemid of morulae $(n=6)$, unexpanded blastocysts $(n=12)$ and expanded blastocysts $(n=16)$ were compared. A trend in the mitotic activity was observed, with the morulae tending to have the lowest mitotic indices; this was not statistically significant $(P=0.26)$.

\section{Discussion}

This study of nuclei counts and mitotic indices in 75 human embryos after 2-6 days in culture has provided an estimate of the rate of cell proliferation in vitro of human embryos throughout the preimplantation period. We conclude that, while the mean doubling time of cells during the first two division cycles is approximately $20 \mathrm{~h}$, the mean duration of the cell cycle of subsequent divisions is $31 \mathrm{~h}$. The rate of nuclear replication is linear on a $\log$ scale between day 2 and day 6.

In the human preimplantation embryo, the rate of nuclear replication can only be taken as an estimate of the rate of cell division, since there is a high incidence of multinucleated blastomeres at all stages of development (Lopata et al., 1983; Plachot et al., 1986; Tesarik et al., 1987; Winston et al., 1991). Murray et al. (1986) and Winston et al. (1991) suggest that the presence of multinucleated cells in blastocysts represents giant cell formation in the trophoblast.

Previous estimates of the mean cell doubling time of in vitro cultured human embryos have ranged from 11.9 to $24 \mathrm{~h}$ 

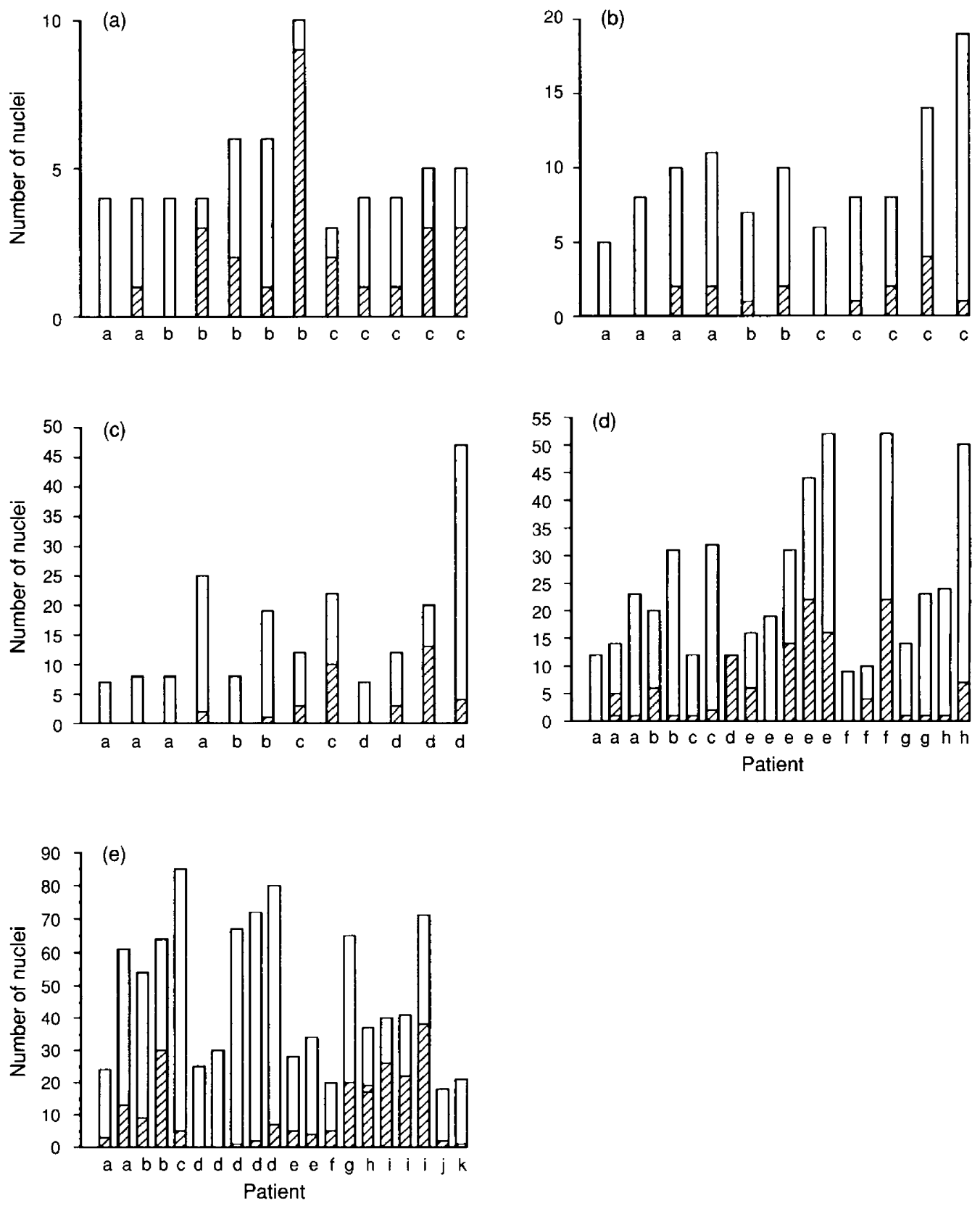

Fig. 2. Number of interphase ( $\square$ ) and mitotic ( $\nabla)$ nuclei per human embryo on (a) day 2 (44-50 h after insemination); (b) day 3 (68-70 h after insemination); (c) day 4 (96-98 h after insemination); (d) day 5 (120-122 h after insemination) and (e) day 6 (144-146 h after insemination). Embryos from individual couples are denoted by the letters $a-k$.

(Edwards et al., 1981; Trounson et al., 1982; Cummins et al., 1986; Hardy et al., 1989). In an analysis of the growth rate of 208 embryos from 22 to $65 \mathrm{~h}$ after insemination, Cummins et al. (1986) found a mean doubling time of $11.9 \mathrm{~h}$. However, it is likely that this value is artificially low because the analysis was based on the assumption that embryos at the morula stage had 64 cells; the evidence from the present study and that of Hardy et al. (1989) suggests that embryos containing this number of cells would already have become blastocysts. From a study of nuclei counts of human blastocysts on days 5, 6 and 7. Hardy et al. (1989) conclude that the mean doubling time of human embryos between the one cell and the blastocyst stage is $24 \mathrm{~h}$.

The results of the present study show that the mean number of cell replication cycles completed by day $2(48-50 \mathrm{~h}$ after insemination) was 2.23 . It is not possible to determine accurately the mean doubling time for the first two division cycles because the exact time at which fertilization occurred is not known. However, Trounson et al. (1981) reported that the first cleavage division is not observed until at least $27 \mathrm{~h}$ after insemination. On this basis, the time taken to complete the second cell division cycle in our study is $17-19 \mathrm{~h}$, which is in 

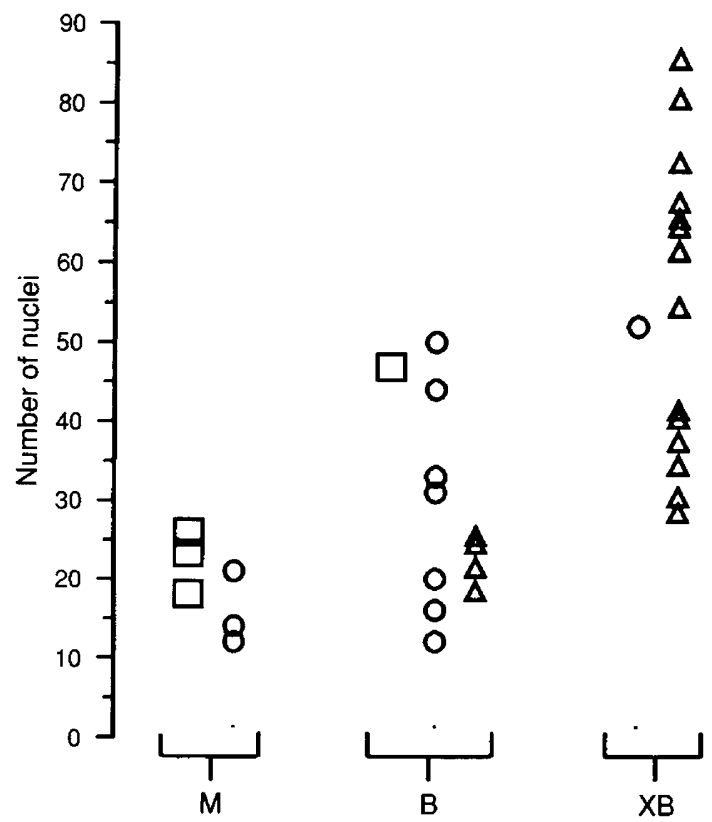

Fig. 3. Correlation between the number of nuclei and different stages of development in humans of later preimplantation development on $(\square)$ day 4 ; $(O)$ day 5 and $(\triangle)$ day 6. M: morula $(n=6)$; B: blastocyst $(n=12)$; XB: expanded blastocyst $(n=16)$.

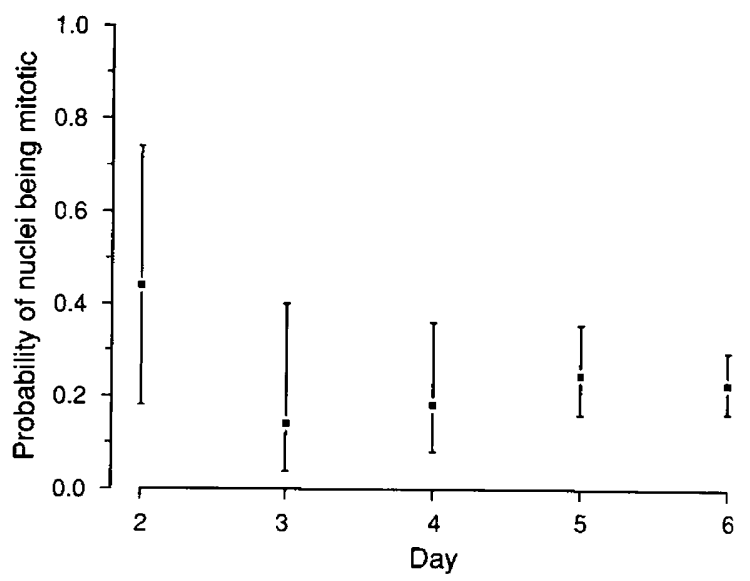

Fig. 4. Probability of human nuclei being mitotic after exposure for $16 \mathrm{~h}$ to colcemid at the end of $2,3,4,5$ and 6 days of culture in vitro. Vertical bars represent $95 \%$ confidence intervals.

close agreement with other reports of the mean doubling times during the early division cycles of $18 \mathrm{~h}$ (Trounson et al., 1982) and $15.8 \mathrm{~h}$ (Edwards et al., 1981). In the present study, the overall doubling time between day 2 and day 6 was $31.6 \mathrm{~h}$. It would therefore appear that the rate of human embryonic ceil proliferation in vitro slows down between the second and third replication cycle, with the mean time taken to complete a division cycle increasing by almost $50 \%$ after the first two cycles.

The timing of a reduction in the rate of cell proliferation coincides with the reported timing of activation of the human embryonic genome (Braude et al., 1988). Artley et al. (1992) were unable to demonstrate a direct causal relationship between cleavage arrest and the onset of gene expression in

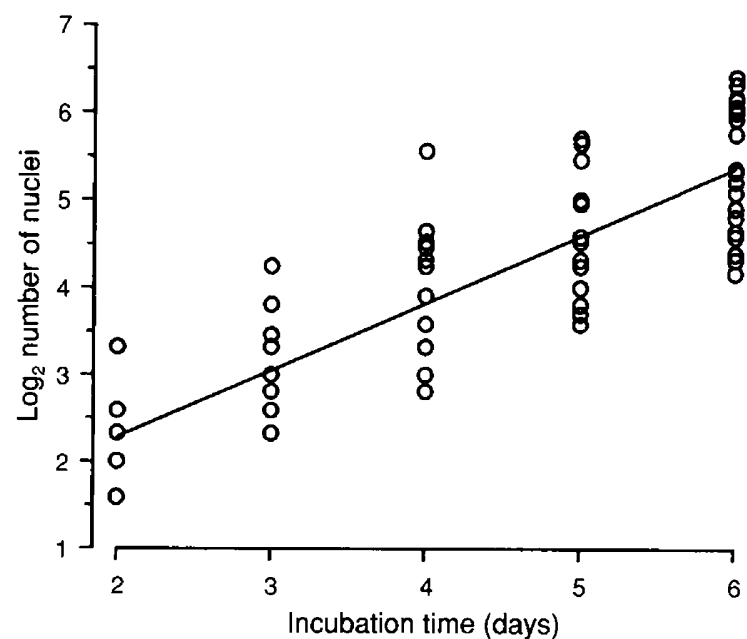

Fig. 5. Total number of nuclei of 75 embryos after 2-6 days of culture in vitro.

human embryos. However, a number of models for the regulation of mitogenic activity during embryogenesis in nonmammalian species have proposed that the transition from regulation of cell function by maternal gene products to embryonic genome expression is related to the observed reduction in the rate of cell proliferation between the early and late division cycles (O'Farrell et al., 1989; Hartwell and Weinert, 1989; Obeyesekere et al., 1994). It is conceivable that, once the embryonic genome assumes control, human embryos require an extracellular stimulus to promote mitosis and that the absence of such a factor in the culture medium may be responsible for the generally low mitotic activity reported here.

With the exception of day 2 , the mitotic indices were lower than would be expected from the calculations based on numbers of cells on each day. This may reflect the progressive inability of the culture medium to support development owing to increased metabolic requirements of the embryos or build up of inhibitory factors in the culture environment. Studies on a number of species have shown that embryos cultured in vitro grow at a slower rate than do embryos in vivo (Streffer $e$ t al. 1980; Papaioannou and Ebert, 1988). Such a comparison is not possible in humans because of the paucity of data on development in vivo. Steffer et al. (1980) suggest that there may be a physiological basis for the observed reduction of mitotic activity in vitro, because mouse embryos developed in vivo show a reduction in the rate of replication after the third cell cycle; this is attributed to an extended G2 phase.

Despite the fact that degenerating and morphologically abnormal embryos were excluded from the present analysis either because they were lost in the fixative or because the extent of nuclear degeneration did not permit an accurate estimate of the number of nuclei, it is possible that some arrested embryos were included in the analysis and therefore contributed to the overall low rate of mitosis. As the specific stage of the cycle of those cells with interphase nuclei was not determined, we cannot state confidently whether these cells had stopped cycling. Furthermore, the finding that a number of embryos, particularly at the later stages of development, had low total nuclei counts but high mitotic indices cautions against the use of arbitrary definitions of developmental arrest. 
Hardy et al. (1989) report a high rate of cell death, as evidenced by nuclear degeneration. While a substantial proportion of fixed embryos were excluded from our analysis because of extensive nuclear degeneration, this was not a feature of the embryos that were analysed.

It could be argued that either the concentration of colcemid used ( $\left.1.25 \mathrm{~mol} \mathrm{l}^{-1}\right)$ was not sufficiently potent to arrest the cells in metaphase or a nonspecific toxic effect inhibited progression through the cell cycle to the metaphase stage. Siracusa et al. (1980) found that a concentration of $3 \mathrm{~mol} \mathrm{l}^{-1}$ was effective in inhibiting cleavage of mouse embryos and that this effect was reversible, indicating low toxicity at this concentration. Copp (1978) injected mice with $60 \mu \mathrm{g}$ colcemid and found no difference between the dead cell indices of colcemid-treated and of control embryos.

The wide range in the number of nuclei of blastocysts of equivalent chronological age is in agreement with the findings of Hardy et al. (1989), Winston et al. (1991) and Dorkas et al. (1993). In the present study, we found that there was a degree of overlap in the nuclei counts of cleavage stage, morula and blastocyst stage embryos; for example a day 5 embryo was classified as an uncompacted 12-16 nuclei cell but was found to contain 19 nuclei, while a newly formed blastocyst was found to contain only 12 nuclei with no evidence of nuclear degeneration. This suggests that control of compaction and cavitation in human embryos is independent of the number of nuclear replications. The work of Dean and Rossant (1984) shows that inhibition of DNA replication in mouse embryos does not prevent the onset of cavitation. In the light of the proposal by Smith and McLaren (1977) that the nucleocytoplasmic volume ratio may be responsible for the control of blastocoel formation, it is worth noting that the uncompacted embryo with 19 nuclei had a larger cytoplasmic volume (approximately 33\% more) than did other embryos from the same cohort.

Finally, there is wide variation in the number of cells of embryos of equivalent chronological ages and stages of development; our results demonstrate that this was due in part to a significant difference $(P=0.02)$ in the replication rate of embryos from different subjects. It should also be remembered that up to three of the fastest growing embryos from each cohort had been selected for embryo transfer. These points illustrate the difficulties encountered in the study of human embryogenesis. Much larger studies are needed to overcome such difficulties if significant results are to be produced.

\section{References}

Artley JK, Braude PR and Johnson MH (1992) Gene activity and cleavage arrest in human pre-embryos Human Reproduction 7 1014-1021

Braude P, Bolton V and Moore S (1988) Human gene expression first occurs between the four and eight cell stages of preimplantation development Nature 332 459-461

Copp AJ (1978) Interaction between inner cell mass and trophectoderm of the mouse blastocyst Journal of Embryology and Experimental Morphology 48 $109-125$

Cummins JM, Breen TM, Harrison KL, Shaw JM and Wilson LM (1986) A formula for scoring human embryo growth rates in in vitro fertilisation: its value in predicting pregnancy and in comparison with visual estimates of embryo quality Journal of In Vitro Fertilisation and Embryo Transfer 3 284-295

Dean WL and Rossant J (1984) Effect of delaying DNA replication on blastocyst formation in the mouse Differentiation 26 134-137

Dorkas A, Sargent IL and Barlow DH (1993) Human blastocyst grading: an indicator of developmental potential? Human Reproduction 8 2119-2127

Edwards RG, Purdy JM, Steptoe PC and Walters DE (1981) The growth of human preimplantation embryos in vitro American Journal of Obstetrics and Gynecology 141 408-416

Hardy K, Handyside AH and Winston RML (1989) The human blastocyst: cell number, death and allocation during late preimplantation development in vitro Development 107 597-604

Hartwell LH and Weinert TA (1989) Checkpoints: controls that ensure the order of cell cycle events Science 246 629-634

Johnson MH and Ziomek CA (1982) Cell subpopulations in the late morula and early blastocyst of the mouse Developmental Biology 91 431-439

Lopata A, Kohlman D and Johnston I (1983) The fine structure of normal and abnormal embryos developed in culture. In Fertilisation of the Human Egg In Vitro pp 189-210 Eds HM Beier and HR Lindner. Springer-Verlag, Berlin

MacQueen HA and Johnson MH (1983) The fifth cell cycle of the mouse embryo is longer for smaller cells than for larger cells Journal of Embryology and Experimental Morphology 77 297-308

Margalioth EJ, Taney FH, Cooper GW, Scholl GM and Rosenfield DL (1993) Correlations between serum oestradiol level on the day of hCG and the speed of embryonic development in in vitro fertilization Human Reproduction $8752-754$

Murray JD, Moran C, Boland MP, Nancarrow CD, Sutton R, Hoskinson RM and Scaramuzzi RJ (1986) Polyploid cells in blastocysts and early fetuses from Australian Merino sheep Journal of Reproduction and Fertility 78 439-446

Obeyesekere MN, Tucker SL and Zimmerman SO (1994) A model for regulation of the cell cycle incorporating cyclin A, cyclin B and their complexes Cell Proliferation 27 105-I13

O'Farrell PH, Edgar BA, Lakich D and Lehner CF (1989) Directing cell division during development Science 246 635-640

Papaioannou VE and Ebert KM (1988) The preimplantation pig embryo: cell number and allocation to trophectoderm and inner cell mass of the blastocyst in vivo and in vitro Development 102 793-803

Plachot M, de Grouchy J, Junca AM, Mandelbaum J, Salat-Baroux J and Cohen J (1986) Cytogenetical analysis of human oocytes and embryos in an IVF programme Human Reproduction 1 (Supplement 2) 21

Ponten J, Stein WD and Shall S (1983) A quantitative analysis of human glial cells in culture Journal of Cell Physiology 117 342-352

Siracusa G, Whittingham DG and De Felici M (1980) The effect of microtubuleand microfilament-disrupting drugs on preimplantation mouse embryos Journal of Embryology and Experimental Morphology $6071-82$

Smith JR and Braunschweiger KI (1979) Growth of human fibroblasts at clonal density. Concordance with results from mass cultures Joumal of Cellular Physiology 98 597-601

Smith R and Mclaren A (1977) Factors affecting the time of formation of the mouse blastocoele Journal of Embryology and Experimental Morphology 41 $79-92$

Staessen C, Camus M, Bollen N, Devroey P and Van Steirteghem AC (1992) The relationship between embryo quality and the occurrence of multiple pregnancies Fertility and Sterility $\mathbf{5 7}$ 626-630

Streffer C, van Beuningen D, Molls M, Zamboglou N and Schultz S (1980) Kinetics of cell proliferation in the pre-implanted mouse embryo in vivo and in vitro Cell and Tissue Kinetics 13 135-143

Tesarik J, Kopecny V, Plachot M and Mandelbaum J (1987) Ultrastructural and autoradiographic observations on multinucleated blastomeres of human cleaving embryos obtained by in vitro fertilisation Human Reproduction 2 $127-136$

Trounson AO, Mohr LR, Wood C and Leeton JF (1982) Effect of delayed insemination on in vitro fertilisation, culture and transfer of human embryos Journal of Reproduction and Fertility 64 285-294

Winston NJ, Braude PR, Pickering SJ, George MA, Cant A, Currie J and Johnson MH (1991) The incidence of abnormal morphology and nucleocytoplasmic ratios in 2, 3 and 5 day human pre-embryos Human Reproduction 6 17-24 